\title{
Perspectives on using laser fluorescence spectroscopy in chronological skin ageing assessment
}

Mikhail Makmatov-Rys ${ }^{1 凶}$, Irina Raznitsyna ${ }^{1}, Y_{\text {Ylia Chursinova }}{ }^{1}$, Daria Mosalskaya ${ }^{1}$, Dmitry Rogatkin ${ }^{1}$, Alexey Zulkarnaev $^{1}$, Anton Molochkov ${ }^{1}$, Dmitry Kulikov ${ }^{1}$

${ }^{1}$ M. F. Vladimirsky Moscow Regional Research and Clinical Institute, Moscow, Russia.

\begin{abstract}
Introduction: Laser fluorescence spectroscopy (LFS) is a potential tool for diagnosing pathological skin processes such as fibrosis, hypoxia, and inflammation. This article describes the results of the non-invasive assessment of skin autofluorescence for animals of different ages.

Methods: The study was performed on outbred white male mice $(n=14)$. Fluorescence spectra were measured using the multifunctional device LAKK-M (SPE Lazma, Moscow, Russia), which implements LFS in vivo. The method is to record the fluorescence spectrum of a tissue after laser irradiation at a wavelength of $\lambda_{\mathrm{e}}$ excites its fluorescence. The wavelength $\lambda_{\mathrm{e}}$ is selected corresponding to specific fluorophore properties. To excite the fluorescence, the wavelength $\lambda_{e}=535 \mathrm{~nm}$ was used, which made it possible to estimate the fluorescence intensity of the lipofuscin.

Results: Increased autofluorescence of lipofuscin in the green waveband was detected in animals in the older age group compared to the younger group using LFS. It is important to note that the autofluorescence spectra of lipofuscin are in superposition with the spectra of other fluorophores and that the autofluorescence spectra of lipofuscin overlap the spectra of other fluorophores such as porphyrins. This may affect the interpretation of LFS data.
\end{abstract}

Conclusions: Adjustment of animal age is necessary for the optical assessment of pathological processes using LFS.

Keywords: fluorescence, spectroscopy, non-invasive, diagnostics, in vivo, lipofuscin, skin ageing

Received: 10 November 2019 | Returned for modification: 6 March 2020 | Accepted: 30 March 2020

\section{Introduction}

Skin ageing is a complex process that is affected by internal and external factors. Many studies have been conducted to determine the key molecular mechanisms of skin ageing, as well as methods for objective non-invasive evaluation of this process. It has been shown that one of the most important markers of skin ageing, along with melanin, is the pigment lipofuscin (1).

Lipofuscin is a glycolipoprotein dispersed in the cytoplasm of the cells of all organs and tissues in the form of small yellowishbrown clumps, usually concentrated around the nucleus. The protein portion of lipofuscin is represented by oxidized and crosslinked protein chains, which are difficult for enzymes to break down. The formation and accumulation of lipofuscin contributes to oxidative stress, with a significant part of the pigment deposited in the lysosomes themselves (2). It has been suggested that the formation of lipofuscin is a result of a decrease in lysosomal enzyme activity, mainly cysteine proteases, such as cathepsin B, $\mathrm{H}$, and L (3). According to some authors, lipofuscin and lipofuscinlike compounds should be considered a set of undigested cellular components formed as a result of the phagocytosis and autophagy processes and accumulated as endocytoplasmic granules in both physiological and pathological conditions (4).

The accumulation of lipofuscin in various cell types occurs during ageing of the organism, as well as in many pathological conditions; for example, in diseases associated with atrophy of organs. Its concentration is usually higher in non-dividing cells (e.g., neurons and cells of the skeletal and cardiac muscle tissue) $(1,5)$. For example, during ageing, its accumulation is normally observed in the liver, as well as in cells of the central nervous system, where the formation of lipofuscin, lipofuscin-like lipopigments, and ceroids can be induced by oxidative stress or can be a response to certain lysosomal accumulation diseases (e.g., Batten disease or neuronal ceroid lipofuscinosis). The effects of a number of physical and chemical toxic factors, such as radiation, cisplatin, lead, and mercury, can also trigger lipofuscin formation, both in natural and experimentally modeled conditions. In addition, lipofuscin granules can be detected in a number of tumor and non-tumor pathological conditions: pancreatic neoplasms, non-choroidal melanomas, breast carcinomas, pigmented eyelid cysts, and retinal degenerative diseases $(4,6)$.

It is known that lipofuscin has autofluorescent properties. The fluorescence of this compound can be observed in a wide spectral range, with the spectral shape and amplitude of the fluorescent signal depending on its variability (proteins, lipids, and carotenoids), the number of cross-links, and the degree of oxidation of these heterogeneous compounds, but mainly on the concentration of lipofuscin in tissues, and therefore on the degree or activity of cell ageing (6).

The lipofuscin fluorescence waveband overlaps with those for the majority of endogenous fluorophores, which fluorescence diagnostics is based on. Therefore, estimating the influence of lipofuscin content as a marker of skin ageing on the results of LFS is an important consideration in developing new diagnostic methods. Thereby, the purpose of this study was to compare and analyze the results of LFS for animals belonging to different age groups. 


\section{Methods}

\section{Animals}

The study was performed on outbred white male mice, weighing 25 to 35 grams, $n=14$. The animals were kept in vivarium-standard conditions in 14 -hour natural light at 21 to $23{ }^{\circ} \mathrm{C}$ and 50 to $65 \%$ humidity. They received balanced granulated feed that did not include fluorophores and they had unlimited access to water. The experiment was conducted in compliance with the welfare of animals used in experiments (Declaration of Helsinki, EU Directive $86 / 609 /$ EEC on the protection of animals used in experiments, and European Convention for the Protection of Vertebrate Animals Used for Experimental and other Scientific Purposes [ETS 123] Strasbourg, 1986).

The animals were divided into two groups: 6-week-old mice (young, $n=7$ ) and 1-year-old mice (old, $n=7$ ). The mouse fur was removed in the dorsal region (using Veet depilatory cream) 48 hours prior to the optical measurements.

\section{Optical methods}

All optical measurements were performed using the dorsal skin of the ICR mice. Fluorescence spectra were measured using the multifunctional device LAKK-M (SPE Lazma, Moscow, Russia; http:// www.lazma.ru/eng/index.php), which implements LFS in vivo. The method is to record the fluorescence spectrum of a tissue after the exciting laser irradiates it at the wavelength corresponding to the maximum absorption by the specific fluorophore. Low-power continuous laser radiation (less than $10 \mathrm{~mW}$ ) is delivered from the laser source to the surface of the object under study by lighting fibers of the fiber-optic probe. Such radiation does not damage the skin. The secondary radiation is delivered by the receiving fiber to the spectrometer. The spectrum detected is reflected on the computer's monitor in real time.

To excite the fluorescence, the wavelength $\lambda_{\mathrm{e}}=535 \mathrm{~nm}$ was used, which made it possible to estimate the fluorescence intensity of the lipofuscin.

To quantify the fluorescence spectrum, the tissue fluorophore content indices $n\left(\lambda_{\mathrm{f}}\right)_{\lambda \mathrm{e}}$ were calculated using formula (7):

$$
\eta\left(\lambda_{f}\right)_{\lambda_{e}}=\frac{I_{f}\left(\lambda_{f}\right)}{I_{f}\left(\lambda_{f}\right)+I_{e}\left(\lambda_{e}\right)}
$$

where $\mathrm{I}_{\mathrm{f}}\left(\lambda_{\mathrm{f}}\right)$ is the fluorescence signal intensity at fluorescence wavelength $\lambda_{\mathrm{f}}\left(\lambda_{\mathrm{f}}=580-590 \mathrm{~nm}\right.$ for lipofuscin $)$ and $\mathrm{I}_{\mathrm{e}}\left(\lambda_{\mathrm{e}}\right)$ is the recorded intensity of the laser radiation back-scattered by the tissue at wavelength $\lambda_{e}$, reduced by the factor of $\beta\left(\beta \approx 10^{\wedge} 3\right)$. The fluorescence spectra were measured and the $n\left(\lambda_{\mathrm{f}}\right)_{\lambda}$ were calculated for five regions of the skin. Furthermore, $n\left(\lambda_{\mathrm{f}}\right)_{\lambda \mathrm{e}}$ were averaged for each animal. LFS measurements and data processing took 15 to 20 minutes per mouse.

\section{Statistical analysis}

The statistical analysis was carried out in Microsoft Excel 2016. The hypothesis for the difference between the two groups was tested by comparison of the arithmetic means and the construction of $95 \%$ confidence intervals for them.

\section{Results}

The results of the lipofuscin endogenous fluorescence study showed an increase in the tissue content index $n\left(\lambda_{\mathrm{f}}\right)_{\lambda_{\mathrm{e}}}$ in the group of old mice (1 year) in comparison with the group of young mice (6 weeks). The differences between the groups were statistically significant for $\mathrm{n}\left(\lambda_{\mathrm{f}}\right)_{\lambda \mathrm{e}}(p<0.005)$ (Fig. 1).

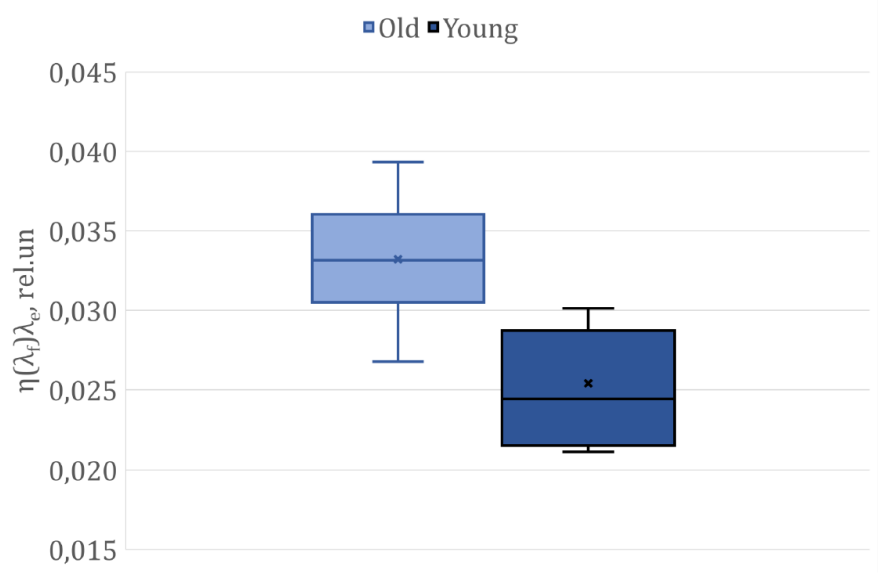

Figure 1 | Tissue lipofuscin content indices $n\left(\lambda_{f}\right)_{\lambda e}, \lambda_{e}=535 n m, \lambda_{f}=580-590$ nm.

\section{Discussion}

Analysis of lipofuscin fluorescence intensity can be used in diagnosing age-related retinal changes, which is confirmed by the experimental and clinical data (8). In addition, some literature has shown the potential of using LFS to estimate skin age (9). Soroka et al. (10) performed fluorescent analysis of human keratinocyte suspensions and found a significant increase in fluorescence intensity at $620 \mathrm{~nm}\left(\lambda_{\mathrm{e}}=480 \mathrm{~nm}\right)$ wavelengths in senescent cell cultures compared to younger cultures. This phenomenon of lipofuscin accumulation corresponded to the results of electron microscopy, confirming the accumulation of dense opaque material localized in the lysosome-like vacuoles in ageing keratinocytes (10). In a clinical study by Moskvin et al. (11), which included 40 women 20 to 55 years old, there was a $28 \%$ increase in the fluorescence contrast ratio of lipofuscin in the skin of women from an older age group $(1.23 \pm 0.08)$ compared with this ratio in young women $(0.89 \pm 0.11)$.

Porphyrin is well known to be fluorescent in the red wavelength range with maximum wavelengths of about 640 and $710 \mathrm{~nm}$. Interestingly, the majority of endogenous fluorophores (NADH, flavins, vitamin A, fatty acids, etc.) fluoresce in the blue-green and yellow spectral regions, which makes it difficult to identify them (6). From this point of view, as a rule porphyrin fluorescence is clearly distinguishable. However, lipofuscin fluoresces in almost the entirely visible wavelength range and therefore contributes to the resulting fluorescence spectrum. As a rule, in a certain wavelength range, the fluorescence of a single fluorophore is investigated, and the contributions of others are neglected. Figure 2 shows an example of the recorded autofluorescence spectrum of the skin of the mice in the young and old age groups. In the skin of the old mice (1 year) the peaks overlap, whereas the contribution of lipofuscin fluorescence to the second peak cannot be 


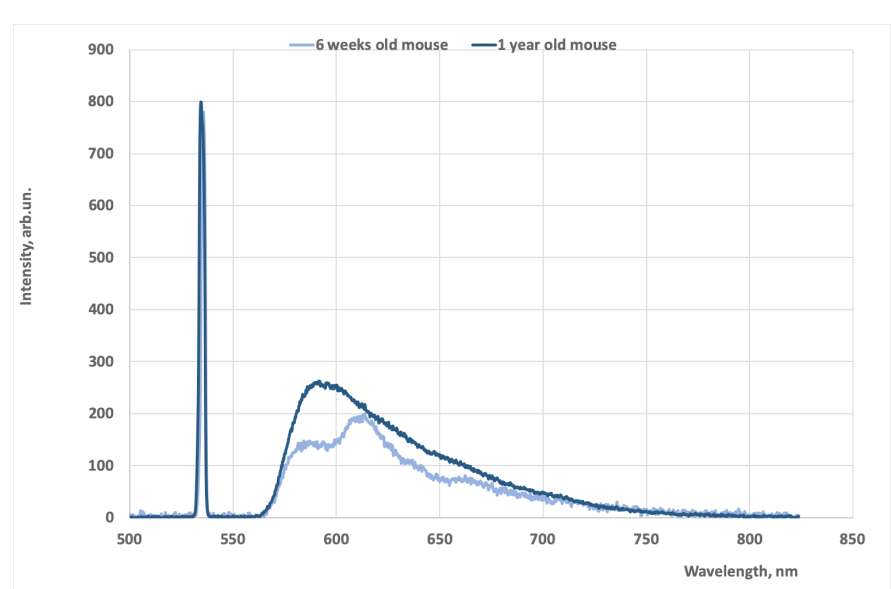

Figure 2 | Examples of registered spectra of young and old mouse skin.

neglected. In addition, many parameters of biological tissue affect the shape of the registered peak of lipofuscin fluorescence, and therefore subtracting this peak from the resulting spectrum is a completely non-trivial task. Thus, the significant fluorescence of lipofuscin observed in the senescent group might interfere with the assessment of the dynamics of metabolic, inflammatory, and hypoxic processes by LFS. In particular, this may affect the results of the dynamic optical observation of inflammation and hypoxia in the development of skin fibrosis (12).

Fluorescence spectroscopy is a convenient method for assessing lipofuscin deposits in tissues, and it can be used to assess skin ageing. Our studies have confirmed previously obtained information that the accumulation of lipofuscin in the skin increases with age. In addition, it is important to make an adjustment for the age of the experimental animal when using LFS in long-term experiments to analyze the fluorescence of other endogenous fluorophores, which are indicators of inflammation and hypoxia.

\section{Acknowledgments}

This study was funded by the Russian Foundation for Basic Research (RFBR) as part of research project 18-02-00564.

\section{References}

1. Skoczyńska A, Budzisz E, Trznadel-Grodzka E, Rotsztejn H. Melanin and lipofuscin as hallmarks of skin aging. Postepy Dermatol Alergol. 2017;34:97-103.

2. Jung T, Höhn A, Catalgol B, Grune T. Age-related differences in oxidative protein-damage in young and senescent fibroblasts. Arch Biochem Biophys. 2009;483:127-35.

3. Schallreuter KU, Kothari S, Chavan B, Spencer JD. Regulation of melanogenesis-controversies and new concepts. Exp Dermatol. 2008;17:395-404.

4. Di Guardo G. Lipofuscin, lipofuscin-like pigments and autofluorescence. Eur J Histochem. 2015;59:2485.

5. Rinnerthaler M, Bischof J, Streubel M, Trost A, Richter K. Oxidative stress in aging human skin. Biomolecules. 2015;5:545-89.

6. Croce AC, Bottiroli G. Autofluorescence spectroscopy and imaging: a tool for biomedical research and diagnosis. Eur J Histochem. 2014;58:2461.

7. Petritskaya EN, Kulikov DA, Rogatkin DA, Guseva IA, Kulikova PA. Use of fluorescence spectroscopy for diagnosis of hypoxia and inflammatory processes in tissue. J Opt Technol. 2015;82:810-4.

8. Sparrow JR, Blonska A, Flynn E, Duncker T, Greenberg JP, Secondi R, et al. Quantitative fundus autofluorescence in mice: correlation with HPLC quantitation of RPE lipofuscin and measurement of retina outer nuclear layer thickness. Invest Ophthalmol Vis Sci. 2013;54:2812-20.
9. Kollias N, Gillies R, Moran M, Kochevar IE, Anderson RR. Endogenous skin fluorescence includes bands that may serve as quantitative markers of aging and photoaging. J Invest Dermatol. 1998;111:776-80.

10. Soroka $Y$, Ma'or Z, Leshem Y, Verochovsky L, Neuman R, Brégégère FM, et al. Aged keratinocyte phenotyping: morphology, biochemical markers and effects of Dead Sea minerals. Exp Gerontol. 2008;43:947-57.

11. Moskvin SV, Antipov EV, Zarubina EG, Ryazanova EA. Vliyanie lazeroforeza na pokazateli fluorescencii, lipofuscina, kollagena i elastina v kozhe lica [Laserphoresis influence on fluorescence, lipofuscin, collagen and elastin levels in face skin]. Vestnik esteticheskoj mediciny. 2011;10:73-8. Russian.

12. Chursinova YV, Kulikov DA, Rogatkin DA, Raznitsyna IA, Mosalskaya DV, Bobrov MA, et al. Lazernaya fluorescentnaya spektroskopiya i opticheskaya tkanevaya oksimetriya $v$ diagnostike fibroza kozhi [Laser fluorescence spectroscopy and optical tissue oximetry in the diagnosis of skin fibrosis]. Biomedical Photonics. 2019;8:38-45. Russian. 